\title{
Impact of pre-brachytherapy magnetic resonance imaging on dose-volume histogram of locally advanced cervical cancer patients treated with radiotherapy including high-dose-rate brachytherapy
}

\author{
Keiko Nemoto Murofushi, MD, PhD, Toshiki Ishida, MD, Keiichiro Baba, MD, Kenji Kawakita, BA, \\ Tsukasa Saida Sasaki, MD, PhD, Toshiyuki Okumura, MD, PhD, Toyomi Sato, MD, PhD, Hideyuki Sakurai, MD, PhD \\ Department of Radiation Oncology and Proton Medical Research Center, Faculty of Medicine, University of Tsukuba, Tsukuba, Ibaraki, Japan
}

\begin{abstract}
Purpose: This study investigated the suitability of brachytherapy technique selection based on pre-brachytherapy magnetic resonance imaging (MRI) findings in cervical cancer by evaluating dose-volume histogram parameters.

Material and methods: We retrospectively evaluated data from 61 patients with cervical cancer who underwent pre-brachytherapy MRI within 7 days before their first high-dose-rate brachytherapy treatment, selected according to pre-brachytherapy MRI findings. Combined intracavitary brachytherapy with interstitial techniques (IC/ISBT) or interstitial brachytherapy (ISBT) were favored treatments for poor-responding tumors after concurrent chemoradiotherapy, asymmetrical tumors, bulky parametrial extensions, bulky primary disease, and extensive paravaginal or distal vaginal involvement. Intracavitary brachytherapy (ICBT) was the preferred treatment for small tumors without extensive involvement of the vagina and parametrium.

Results: The median tumor size was $58 \mathrm{~mm}$ on pre-treatment MRI and $38 \mathrm{~mm}$ on pre-brachytherapy MRI. On pre-brachytherapy MRI, 13 patients had a tumor with severe vaginal invasion, 15 patients presented with an asymmetrical bulky tumor, and 4 patients had bulky residual tumors. IC/ISBT or ISBT were administered to 26 patients (43\%). Median equivalent dose in $2 \mathrm{~Gy}$ fractions of clinical target volume $\mathrm{D}_{90}$ was $70.8 \mathrm{~Gy}$ for all patients. Median clinical target volume $\mathrm{D}_{90}$ in each brachytherapy session exceeded the prescribed dose in both patients treated with ICBT and IC/ISBT or ISBT. Median equivalent dose in $2 \mathrm{~Gy}$ fractions of $\mathrm{D}_{2 \mathrm{cc}}$ to the bladder, sigmoid colon, and rectum was 69.5, 52.0, and 58.4 Gy, respectively. All cases remained within the doses recommended for organs at risk.

Conclusions: Pre-brachytherapy MRI may be helpful in selecting suitable candidates for each type of brachytherapy and deliver the recommended doses to the tumor and organs at risk, particularly in cases with large tumors, poor response to concurrent chemoradiotherapy, asymmetrical tumors, severe vaginal invasion, extensive parametrial invasion, and/or corpus invasion.
\end{abstract}

J Contemp Brachytherapy 2021; 13, 1: 32-38 DOI: https://doi.org/10.5114/jcb.2021.103584

Key words: cervical cancer, image-guided adaptive brachytherapy, intracavitary brachytherapy, interstitial brachytherapy, dose-volume histogram.

\section{Purpose}

For early-stage cervical cancer patients, radical radiotherapy with intracavitary brachytherapy (ICBT) delivering the prescribed dose to point A using Manchester planning methods could achieve a local control (LC) rate of $80-90 \%$ [1,2]. However, the LC remains disappointing in cases with bulky and extensive tumors, as ICBT does not administer an adequate dose to the whole tumor $[3,4]$. In such complex cases, both interstitial brachytherapy (ISBT) and combined intracavitary brachytherapy with interstitial techniques (IC/ISBT) can provide irregularly shaped dose distributions, resulting in an improved LC $[5,6,7,8]$. Even though, characteristics of tumors, which most likely are to benefit from ICBT vs. ISBT or IC/ISBT remain unclear, and no study has described these before, the appropriate type of brachytherapy could be selected according to tumor characteristics in pre-brachytherapy magnetic resonance imaging (MRI).

In our previous study, high-dose-rate (HDR) brachytherapy selection based on pre-brachytherapy MRI find-
Address for correspondence: Keiko Nemoto Murofushi, MD, Department of Radiation Oncology and Proton Medical Research Center, Faculty of Medicine, University of Tsukuba, 1-1-1 Tennodai, Tsukuba, Ibaraki, 305-8576, Japan, phone: +81-29-853-7100, fax: +81-29-853-7102,

e-mail: murofushi@pmrc.tsukuba.ac.jp
Received: 07.05 .2020

Accepted: 06.11.2020

Published: 18.02.2021 
ings has achieved an LC rate of up to $90 \%$ among patients with locally advanced cervical cancer $[9,10]$. Furthermore, LC among patients treated with unsuitable brachytherapy, i.e., brachytherapy other than that selected based on pre-brachytherapy MRI findings, was worse than that among patients treated with appropriate brachytherapy $(p<0.01)$ [10]. However, dose-volume-histogram (DVH) data for the patients treated with ICBT was lacking, as ICBT was performed based on two-dimensional treatment planning. In this study, all types of brachytherapy were planned based on computed tomography (CT); thus, this study aimed to analyze the DVH parameters of three-dimensional ICBT vs. ISBT or IC/ISBT to confirm the appropriate suitability of HDR brachytherapy selection according to pre-brachytherapy MRI findings.

\section{Material and methods}

\section{Eligible patients}

Between June 2017 and April 2019, 63 patients with locally advanced cervical cancer received definitive HDR brachytherapy. We included patients as per the following criteria: 1) both of pre-treatment and pre-brachytherapy MRI were performed, 2) type of brachytherapy selected
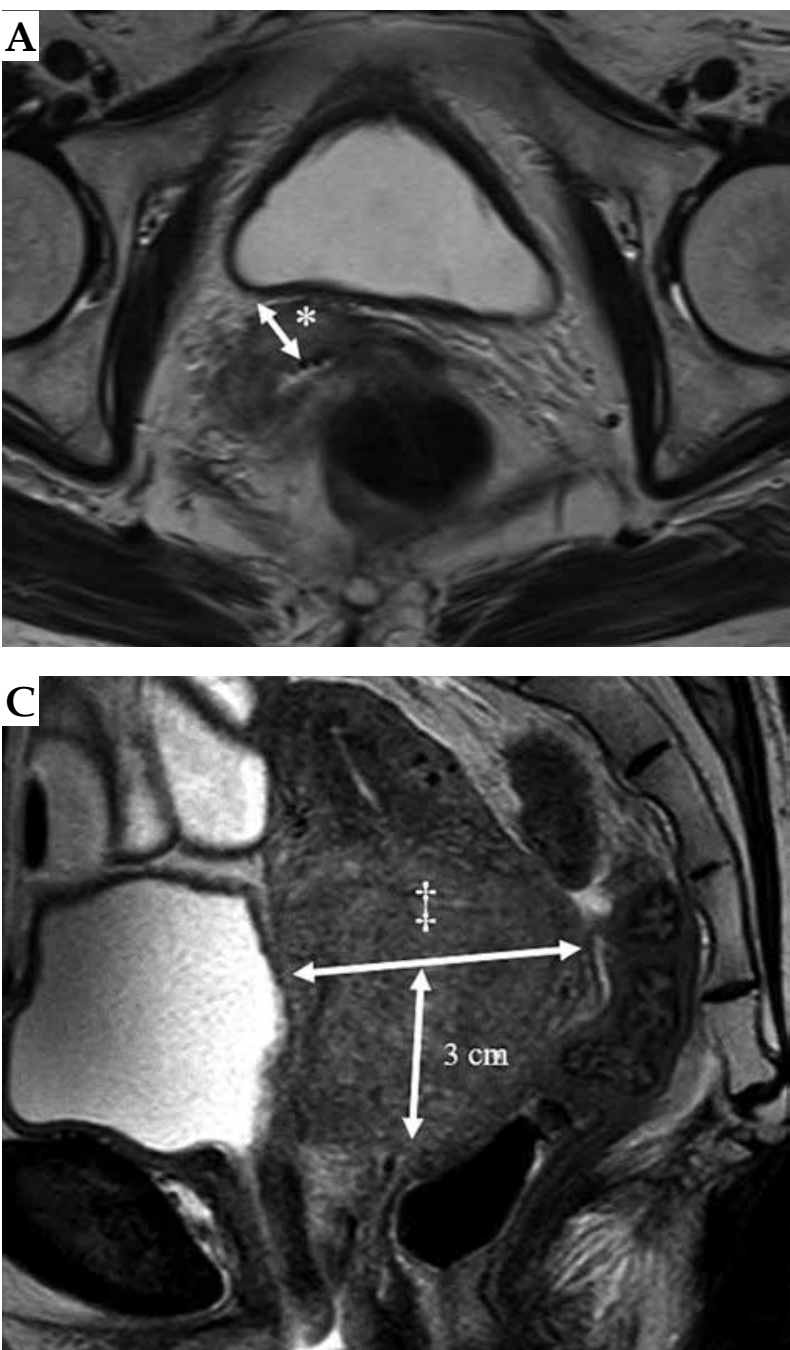

according to pre-brachytherapy MRI findings, 3) the Eastern Cooperative Oncology Group performance status of $0-1,4)$ no history of pelvic irradiation. Finally, this retrospective study evaluated data of 61 patients. The study's protocol was approved by our institutional ethics committee.

\section{MRI examinations}

Pre-brachytherapy MRIs were obtained within 7 days before patients' first HDR brachytherapy. Hyoscine butylbromide (Buscopan ${ }^{\circledR}$; Sanofi, France) was injected intramuscularly to all patients to reduce motion artifact by bowel peristalsis. Pre-treatment and pre-brachytherapy MRI examinations were performed with a 3T system (Ingenia, Philips Medical Systems, Best, The Netherlands), with a phased-array body coil of 32 channels. The field of view was $28 \mathrm{~cm}$, with at least a $256 \times 256$ matrix. The slice thickness was $3 \mathrm{~mm}$, with a $0.3-\mathrm{mm}$ intersection gap. Sagittal T2-weighted images were perpendicular to the uterine long axis with TR/TE $1400 / 110 \mathrm{msec}$, axial T2-weighted images were perpendicular to the uterine short axis with TR/TE 6746/110 $\mathrm{msec}$, and a 24-echo train length was obtained in all cases.

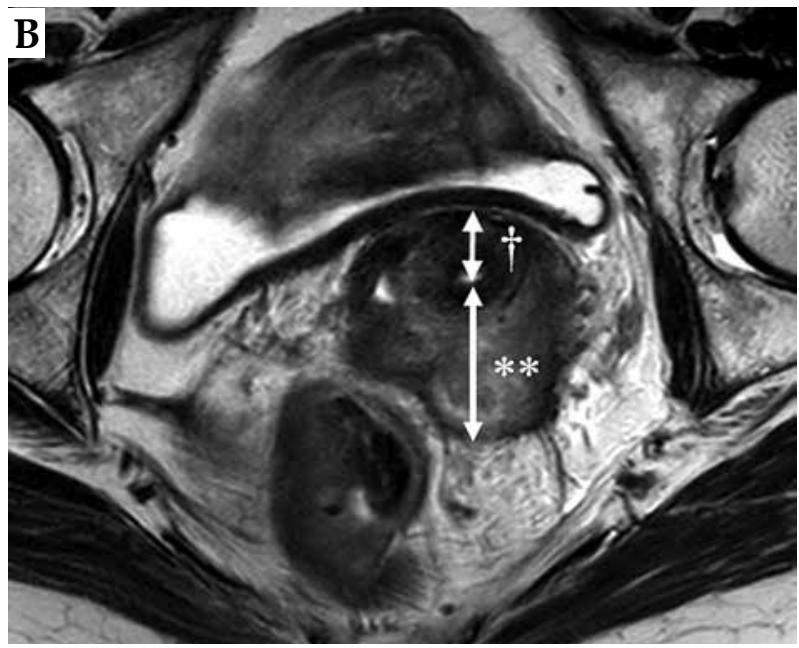

Fig. 1. Typical magnetic resonance imaging findings. Pre-brachytherapy magnetic resonance imaging findings for a tumor with $>5 \mathrm{~mm}$ of vaginal invasion (A), an asymmetrical tumor (B), and a bulky barrel-shaped tumor (C) *thickness of the vaginal invasion, ** distance between the cervical tube and tumor's external edge, + distance between the cervical tube and tumor's external edge or cervix, $¥$ tumor width at $3 \mathrm{~cm}$ above the external os 


\section{Selection of brachytherapy type}

Our previous studies mention more details about selection of brachytherapy type $[9,10]$. The residual tumor was defined as the high T2WI area on the pre-brachytherapy MRI. IC/ISBT or ISBT were selected for cases with vaginal invasion, bulky asymmetrical tumors, and bulky barrel-shaped tumors based on pre-brachytherapy MRI scans (Figure 1). Vaginal invasion was defined as present in cases with vaginal invasion thicker than $5 \mathrm{~mm}\left({ }^{*}\right.$ in Figure 1A). Bulky asymmetrical tumors were defined as a tumor defined by two criteria: distance between cervical canal and distal tumor edge (** in Figure 1B) was larger than $2 \mathrm{~cm}$, and [distance between cervical canal and distal tumor edge, ${ }^{* *}$ ] minus [contralateral tumor distance, $\dagger$ ] (Figure 1B) was $1 \mathrm{~cm}$ or larger. Bulky barrel-shaped tumors were defined as invading the uterine fundus or with a width of $\geq 5 \mathrm{~cm}$ at $3 \mathrm{~cm}$ above the external os ( $\ddagger$ in Figure 1C) [10]. ICBT was selected for patients without these findings. The above-mentioned tumor classifications were established by both diagnostic radiologist and a radiation oncologist.

\section{Radiotherapy and brachytherapy protocol}

Treatment schedules were divided into two groups, based on the tumor response and extension of the tumor (Figure 2). Good response was defined by tumor shrinkage and/or parametrium invasion mollified after whole-pelvis external beam radiation therapy (EBRT) in the gynecological examination. Poor response was described as an unchanged or grown tumor. Extensive tumor was defined as the tumor, for which further reduction was desired since a prescribed dose could not be administered to high-risk clinical target volume (HRCTV), even by IC/ISBT or ISBT. In the group 1, a total $\mathrm{EQD}_{2}$ of 60-70 Gy was administered to $\mathrm{D}_{90}$ for HR-CTV, and 70-80 Gy was delivered in group 2 .

All patients underwent EBRT and HDR brachytherapy. EBRT was administered using a three-dimensional conformal technique with a linear accelerator (Clinac IX; Varian Medical System, Palo Alto, CA, USA) and a 10-MV photon beam. For the International Federation of Gynecology and Obstetrics (FIGO) stage IB-III cervical cancer patients, whole-pelvis (WP)-EBRT was initially administered at a dose of 30 Gy in 15 fractions using four-field technique; an additional dose of $20 \mathrm{~Gy}$ in 10 fractions was applied with EBRT, with a 3-cm midline block (MB) and anterior-posterior/posterior-anterior technique. For stage IVA, a dose of 40 Gy in 20 fractions with WP-EBRT, and an additional dose of $10 \mathrm{~Gy}$ in 5 fractions with MB were delivered. For the patients with poor response and/or extensive tumors, a dose of 40-50 Gy in 20-25 fractions with WP-EBRT and an additional dose of 0-10 Gy in 0-5 fractions with $\mathrm{MB}$ were administered. In cases where a pelvic lymph node had the shortest diameter of $\geq 1 \mathrm{~cm}$, an EBRT boost dose of 6-10 Gy was applied in 3-5 fractions.

Brachytherapy was performed using iridium-192 $\left({ }^{192} \mathrm{Ir}\right)$ remote after-loading system (MicroSelectron HDRTM; Nucletron, Veenendaal, The Netherlands), and the first HDR brachytherapy treatment was performed within 7 days after midline block insertion. Brachytherapy schedule was decided based on FIGO stage, tumor response, and extension of the tumor as shown in Figure 2. ICBT was administered once per week, at a dose prescribed to $\mathrm{D}_{90}$ for HR-CTV of 20-24 Gy in 4 fractions (Figure 3A). IC/ISBT was used to deliver a dose of 20-24 Gy in 4 fractions (prescribed as $D_{90}$ to HR-CTV) once per week using tandem-ovoid applicators and 1-3 transvaginal needles (Figure 3B). During ISBT, Martinez universal perineal interstitial template was used to deliver a dose of 14-30 Gy in 2-5 fractions on 1-3 consecutive days (prescribed as $\mathrm{D}_{90}$ to HR-CTV) (Figure 3C). CT-based brachytherapy planning was performed using Oncentra version 4.0 (Nucletron) and a bright speed excel CT scanner (General Electric Healthcare, Chicago, IL, USA). Image acquisition was performed with tube voltage of $140 \mathrm{kVp}$, rotation time of $1.0 \mathrm{~s}$, detector configuration of $4 \times 0.5 \mathrm{~mm}$, pitch of 0.75 , a slice thickness of $2.5 \mathrm{~mm}$, display field-of-view of $450 \mathrm{~mm}$, and standard reconstruction kernel. The dose was calculated using the Oncentra system without applying a heterogeneity correction according to the American Association of Physicists in Medicine Task Group 43 protocol [11].

In the guidelines reported by the Groupe Européen de Curietherapie and the European Society for Radiotherapy and Oncology (GEC-ESTRO), HR-CTV includes residual tumor at the time of brachytherapy, whole cervix, and adjacent residual pathologic tissue [12]. In this study, the HR-CTV included the macroscopic residual tumor with high signal intensities on T2-weighted images (T2WI) in the pre-brachytherapy MRI, the tumor extension recognized by gynecologic examination, and the entire cervix. An image diagnostician defined the macroscopic residual tumor detected in the pre-brachytherapy MRI, and a radiation oncologist finally defined HR-CTV based on the findings of a gynecologic examination.

\section{Chemotherapy}

The indications for concurrent chemoradiotherapy (CCRT) were as follow: 1) FIGO stage IIB or higher, 2) cer-

\begin{tabular}{|c|c|c|c|}
\hline \multirow{2}{*}{ The dose of whole-pelvis EBRT } & \multicolumn{2}{|c|}{ Group 1. Good response } & \multirow{3}{*}{$\begin{array}{c}\text { Group 2. Poor response } \\
\text { and/or extensive tumor } \\
\begin{array}{c}\text { 40-50 Gy/20-25 fx } \\
+\end{array}\end{array}$} \\
\hline & $30 \mathrm{~Gy} / 15 \mathrm{fx}$ & $40 \mathrm{~Gy} / 20 \mathrm{fx}$ & \\
\hline & + & + & \\
\hline \multirow[t]{2}{*}{ The dose of ICBT or IC/ISBT } & $24 \mathrm{~Gy} / 4 \mathrm{fx}$ & $20 \mathrm{~Gy} / 4 \mathrm{fx}$ & $24 \mathrm{~Gy} / 4 \mathrm{fx}$ \\
\hline & or & or & or \\
\hline ISBT & $30 \mathrm{~Gy} / 5 \mathrm{fx}$ & $24 \mathrm{~Gy} / 4 \mathrm{fx}$ & $14-30 \mathrm{~Gy} / 2-5 \mathrm{fx}$ \\
\hline $\mathrm{EQD}_{2}(\alpha \beta 10)$ & 62-70 Gy & 65-72 Gy & 69.8-80 Gy \\
\hline
\end{tabular}

Fig. 2. Treatment schedule 

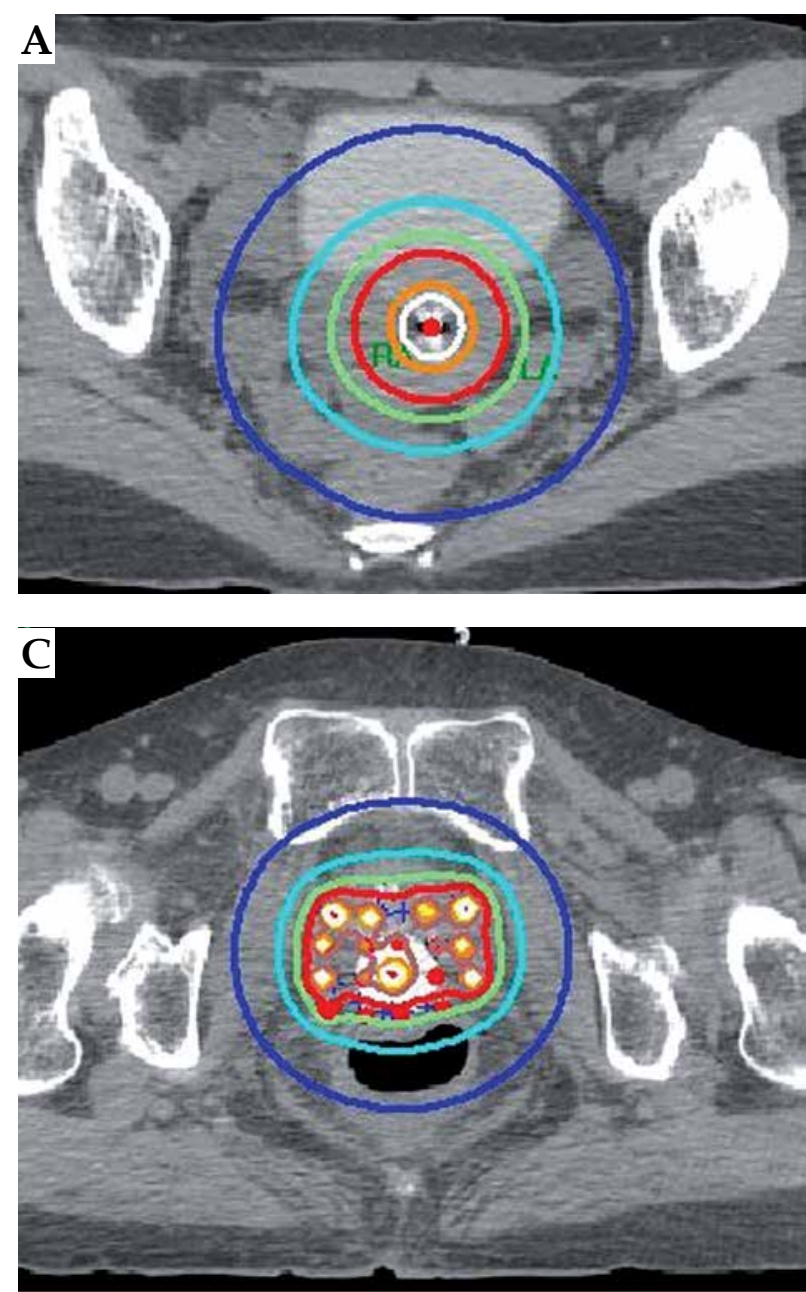

vical tumor size of $4.0 \mathrm{~cm}$ or more, and 3) positive pelvic and/or para-aortic lymph nodes. During the radiotherapy period, patients generally received a weekly regimen of cisplatin $\left(40 \mathrm{mg} / \mathrm{m}^{2}\right)$. CCRT was not performed for patients with insufficient renal function or for those, who were $\geq 75$ years old. Ultimately, seven patients received radiotherapy alone and 54 patients received CCRT.

\section{Statistical analysis}

For the dose summation of brachytherapy plus EBRT before the insertion of $\mathrm{MB}$, the equivalent dose in $2 \mathrm{~Gy}$ fractions $\left(\mathrm{EQD}_{2}\right)$ was calculated, based on the linear-quadratic model [13]. The tumor dose was calculated using an $\alpha / \beta$ ratio of $10 \mathrm{~Gy}$. For calculating the dose-volume parameters of organ at risk (OAR) (i.e., $\left.D_{2.0 c c}\right)$, the $\alpha / \beta$ ratio was assumed to be $3 \mathrm{~Gy}$. The relative proportion was defined using the following formula:

$$
\frac{\text { HR-CTV D }}{\text { prescription dose of brachytherapy }} \times 100(\%) \text {. }
$$

Categorical variables were described as frequency and percentages, and continuous variables were defined using mean, median, and interquartile range (IQR) values. To compare dose of whole-pelvic irradiation, DVH data, and relative proportion of HR-CTV and OARs be-

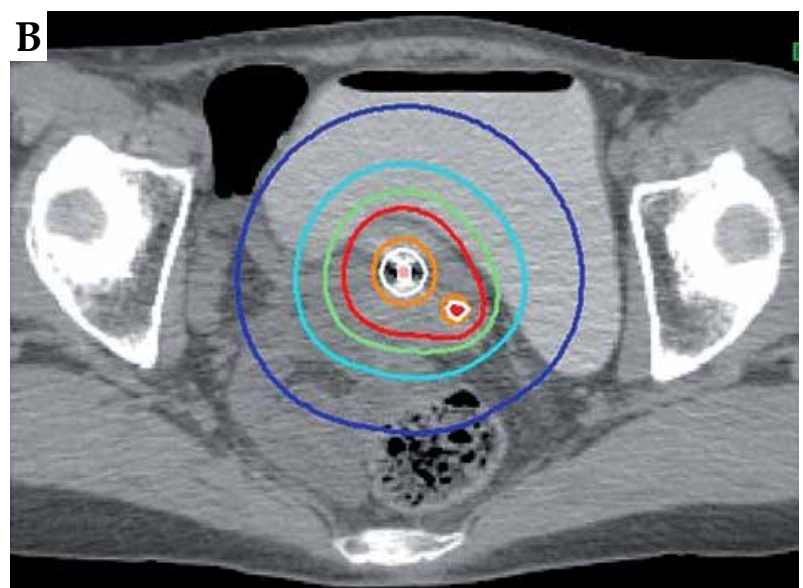

Fig. 3. Dose distributions of brachytherapy. Dose distributions are shown for intracavitary brachytherapy (ICBT) (A), combined intracavitary brachytherapy with interstitial technique (IS/ICBT) (B) and interstitial brachytherapy (ISBT) (C). Blue line - 25\% isodose line, light blue line $50 \%$, green line $-75 \%$, red line - prescribed dose, orange line $-150 \%$, white line $-200 \%$

tween ICBT and ISBT plans, means for continuous variables were compared using Student's t-test if the data were normally distributed; otherwise, Mann-Whitney test was applied. The normality of data was assessed with Kolmogorov-Smirnov test. Statistical significance was defined as $p<0.05$. All statistical analyses were performed using SPSS Base System software program version 24.0.0.0 (SPSS, Chicago, IL, USA) and SAS software version 9.4 (SAS Institute, Cary, NC, USA).

Although, the analyses were not adjusted for multiple comparisons, and given the potential for type I error, our findings should be interpreted as exploratory and descriptive.

\section{Results}

Patients' and tumor characteristics are presented in Table 1 . The median tumor size at pre-brachytherapy MRIs was $38 \mathrm{~mm}$ (range, $0-79 \mathrm{~mm}$ ). In the pre-brachytherapy MRI, 13 patients had a tumor with severe vaginal invasion, 15 presented with an asymmetrical tumor, and 4 had a bulky barrel-shaped tumor. Four patients had an asymmetrical tumor with severe vaginal invasion, and 2 had an asymmetrical tumor with a bulky barrel-shape. Finally, 26 patients $(43 \%$ ) were treated with IC/ISBT (6 patients, $10 \%)$ or ISBT (20 patients, $33 \%)$. The remaining 
Table 1. Patient and tumor characteristics

\section{Parameter}

\begin{tabular}{lc}
\hline Age (years), median (range) & $61(30-88)$ \\
\hline FIGO stage & $1(2 \%)$ \\
\hline IB & $11(18 \%)$ \\
\hline IVA & $39(64 \%)$ \\
\hline Pelvic lymph node metastasis & $10(16 \%)$ \\
\hline Negative & $19(31 \%)$ \\
\hline Positive & $42(69 \%)$ \\
\hline Para-aortic lymph node metastasis & $51(84 \%)$ \\
\hline Negative & $10(16 \%)$ \\
\hline Positive & $53(87 \%)$ \\
\hline Histology & $7(11 \%)$ \\
\hline Squamous cell carcinoma & $1(2 \%)$ \\
\hline Adenocarcinoma & $58(28-108)$ \\
\hline Other & $38(0-79)$ \\
\hline Tumor size (mm), median (range) \\
\hline Pre-treatment MRI
\end{tabular}

FIGO - International Federation of Gynecology and Obstetrics, MRI - magnetic resonance imaging

35 patients $(57 \%)$ received ICBT. Group 1 with good tumor response included 35 patients treated with ICBT, and 15 patients treated with IC/ISBT or ISBT. Group 2 with poor tumor response and/or extensive tumor included 0 and 11 patients, respectively. The median dose of whole-pelvis EBRT for IC/ISBT or ISBT-treated patients (40 Gy) was significantly higher than that for ICBT-treated patients (30 Gy, $p<0.01$ ).

Table 2 shows volume of HR-CTV and DVH parameters. The median $\mathrm{EQD}_{2}$ of HR-CTV $\mathrm{D}_{90}$ was $70.8 \mathrm{~Gy}$ (IQR, 66.8-73.5 Gy) for all patients. The $\mathrm{EQD}_{2}$ of HRCTV $D_{90}$ was significantly higher $(p<0.01)$ in the IC/ ISBT- or ISBT-treated patients than in the ICBT-treated patients, although the median volume of HR-CTV was more than doubled. Figure 4 indicates the relationship between volume of HR-CTV and HR-CTV $D_{90}$. In the patients treated with ICBT, the median $\mathrm{EQD}_{2}$ of $\mathrm{HR}$ CTV $D_{90}$ was 70 Gy (IQR, 67.3-72.2 Gy) and 66.7 (range,
65.0-68.8) for the volume of HR-CTV $\leq 30 \mathrm{cc}$ and HRCTV $>30 \mathrm{cc}$, respectively $(p=0.76)$. In only one patient treated with IS/ICBT, the HR-CTV volume was $30 \mathrm{cc}$ or smaller. There were no significant differences in the $\mathrm{EQD}_{2}$ of $\mathrm{D}_{2 c c}$ to the bladder or sigmoid colon between IC/ISBT- or ISBT- and ICBT-treated patients $(p=0.90$, $p=0.08$, respectively). However, the $\mathrm{EQD}_{2}$ of $\mathrm{D}_{2 c c}$ to the rectum of IC/ISBT- or ISBT-treated patients was significantly higher than that of ICBT-treated patients $(p<0.01)$. Moreover, no patients received doses to the OARs in excess of doses recommended by the American Brachytherapy Society (ABS) (bladder, 90 Gy; sigmoid and rectum, $75 \mathrm{~Gy})$ [14].

The relative proportions of HR-CTV and OARs are demonstrated in Figure 5. In the ICBT-treated patients, the relative proportion of HR-CTV was higher than that of IC/ISBT- or ISBT-treated patients $(p<0.01)$; however, the median relative proportion exceeded $100 \%$ in both treatment groups. The relative proportions of bladder and rectum in the IC/ISBT- or ISBT-treated patients were significantly lower than those in the ICBT-treated patients $(p<0.01, p \leq 0.01$, respectively).

\section{Discussion}

For cervical cancer patients, dose delivered to HRCTV was considered as a surrogate marker for local control $[5,15]$. in this study, the median $\mathrm{EQD}_{2}$ of HR-CTV $\mathrm{D}_{90}$ (70.8 Gy, IQR, 66.8-73.5 Gy) was much lower than that recommended by the Groupe Européen de Curietherapie, the European Society for Radiotherapy and Oncology (GEC-ESTRO), and the ABS $[14,16]$. However, a Japanese protocol with a lower dose over a shorter treatment time has demonstrated comparable outcomes of concurrent chemoradiotherapy delivering an $\mathrm{EQD}_{2}$ of 62-65 Gy to point A comparing with that demonstrated in studies from the United States and European countries [17]. In fact, Murakami et al. have shown that in the Japanese protocol, the dose of 60 Gy or greater for HR-CTV $\mathrm{D}_{90}$ resulted in 3-year LC at $97.3 \%$ [15]. Also, Ohno et al. reported 5-year LC rate of $96 \%$ for FIGO stage IB-IVA cervical cancer patients and in $90 \%$ of them, greater than $60 \mathrm{~Gy}$ was delivered to HR-CTV $\mathrm{D}_{90}$ [18]. In this study, the dose of 60 Gy or greater can be administered to HR-CTV $D_{90}$ for both treatment groups. Therefore, it was estimated that more than $90 \%$ of LC might be obtained for the patients with severe vaginal invasion, bulky parametrial extension, or bulky tumor.

Table 2. Volumes of high-risk clinical target volume and dose-volume histogram parameters

\begin{tabular}{lcccc} 
& All $(N=61)$ & $\begin{array}{c}\text { ICBT } \\
(n=35)\end{array}$ & $\begin{array}{c}\text { IC/ISBT or ISBT } \\
(n=26)\end{array}$ & $P$-value \\
\hline Volume of HR-CTV (cc), median (IQR) & $39.5(25.4-57.5)$ & $28.6(21.7-37.2)$ & $62.7(46.5-79.7)$ & $<0.01^{*}$ \\
\hline HR-CTV D $D_{90}(G y)$ & $70.8(66.8-73.5)$ & $68.8(66.2-71.7)$ & $73.7(70.9-75.1)$ & $<0.01^{* *}$ \\
\hline Bladder $D_{2 c c}(G y)$ & $69.5(66.1-72.0)$ & $68.8(66.3-72.7)$ & $70.0(65.4-71.9)$ & $0.9^{* *}$ \\
\hline Sigmoid $D_{2 c c}(G y)$ & $52.0(48.7-55.4)$ & $50.6(48.7-54.3)$ & $53.3(50.1-57.1)$ & $0.08^{* *}$ \\
\hline Rectum $D_{2 c c}(G y)$ & $58.4(54.6-63.6)$ & $56.8(53.3-58.8)$ & $64.3(59.4-65.5)$ & $<0.01^{* *}$
\end{tabular}

CTV - clinical target volume, HR - high-risk, ICBT - intracavitary brachytherapy, IC/ISBT - combined intracavitary brachytherapy with interstitial technique, $I Q R$ - interquartile range, ISBT - interstitial brachytherapy, "Mann-Whitney U-test, ${ }^{* *}$ Student's t-test 
Most patients treated with IC/ISBT or ISBT, received a dose of 40 Gy or more with WP-EBRT, because this treatment schedule was applied for the patients with FIGO stage IVA, poor response, and/or extensive tumor. Furthermore, the total $\mathrm{EQD}_{2}$ delivered to the patients treated with IS/ICBT or ISBT was higher than that in the ICBT group; however, there was no significant difference in the $\mathrm{EQD}_{2}$ of $\mathrm{D}_{2 \mathrm{cc}}$ to the bladder between two treatment groups, and no patients exceeded the ABS recommended dose [14]. This was probably due to a higher relative proportion of bladder in the patients treated with ICBT. The $E_{2} D_{2}$ of $D_{2 \mathrm{cc}}$ to the rectum in the patients treated with IS/ICBT or ISBT (median, 64.3 Gy, IQR, 59.4-65.5 Gy) was significantly higher than that in patients treated with ICBT (median, 56.8 Gy, IQR, 53.3-58.8 Gy). It had been reported that for patients treated with ICBT or IS/ICBT, mean $\mathrm{EQD}_{2}$ of $\mathrm{D}_{2 \mathrm{cc}}$ to the rectum was 61.8-66.3 Gy [5,19], and $\mathrm{EQD}_{2}$ of $\mathrm{D}_{2 \mathrm{cc}}$ for a $10 \%$ probability for overall rectum grade 2 or more morbidity was $69.5 \mathrm{~Gy}$ [20]. In this study, the $\mathrm{EQD}_{2}$ of $\mathrm{D}_{2 \mathrm{cc}}$ to the rectum in the patients treated with IS/ICBT or ISBT was not considered extreme high, and all cases remained within the doses recommended by the ABS [14]. The reasons for such a significant difference might be higher doses with WP-EBRT and total $\mathrm{EQD}_{2}$ administering to most patients treated with IC/ISBT or ISBT as well as severe vaginal invasion required to include the entire vagina in HR-CTV for 13 patients (50\%), regardless of a lower relative proportion of rectum in the ICBT patients.

Previous studies have shown that IS/ICBT was performed in $4 \%$ to $65 \%$ of patients with locally advanced cervical cancer, where it was able to deliver adequate dose to the entire tumor $[5,18,19]$. The MRI-guided brachytherapy in locally advanced cervical cancer (retroEMBRACE and EMBRACE I) studies had divided participating institutions into IC and IS/IC groups, according to technique of brachytherapy. The IS/IC group included centers performing IS/ICBT consistently in > $20 \%$ of patients. In the retroEMBRACE study, LC improved by $10 \%$ in patients with CTV $\geq 30 \mathrm{cC}$ in the IS/IC group [5]. The EBRACE I study has shown that IS/ICBT was performed in $91 \%$ of patients with parametrium involvement [19]. Jastaniyah et al. reported that IS/ICBT was frequently used in patients with large tumor, tumor with poor response or tumor involvement to distal parametrium, or pelvic wall in MRI at the time of brachytherapy [20]. While the criteria for selecting IS/ICBT technique were continuing to discuss in these studies, IS/ICBT was typically performed in patients with large tumors and extensive parametrial and/or vaginal involvement $[5,18,20,21]$. However, IS/ICBT or ISBT might not be performed for all these tumors in facilities, where IS/ICBT or ISBT were not routinely performed. In a questionnaire-based survey regarding image-guided adaptive brachytherapy, limited number of staff and time were major impediments for performing image-guided adaptive brachytherapy [22]. Under these circumstances, in order to secure the staff and time in advance by selecting appropriate type of brachytherapy before starting the procedure, may lead to more frequently performed IS/ICBT or ISBT, resulting in an improved LC. It was more desirable that an appropriate brachytherapy could

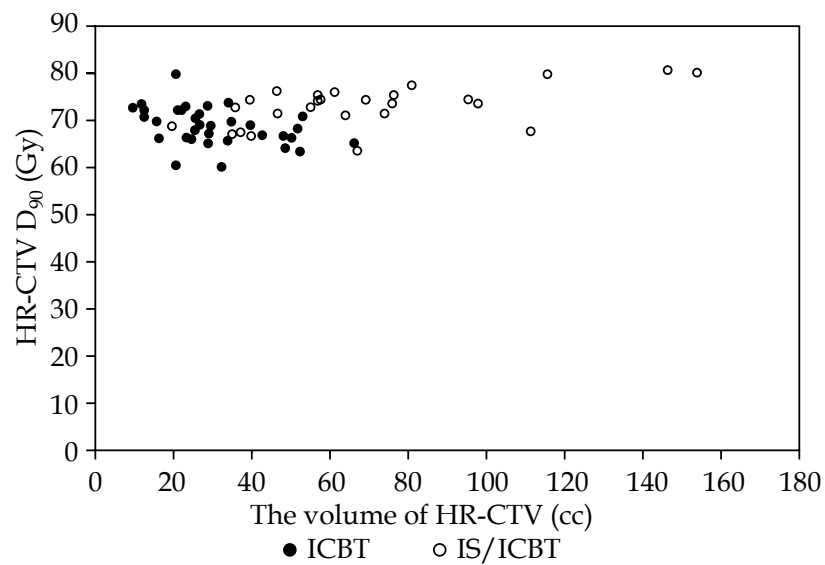

Fig. 4. Relationship between the volume of high-risk clinical target volume (HR-CTV) and clinical target volume (CTV) $\mathrm{D}_{90}$

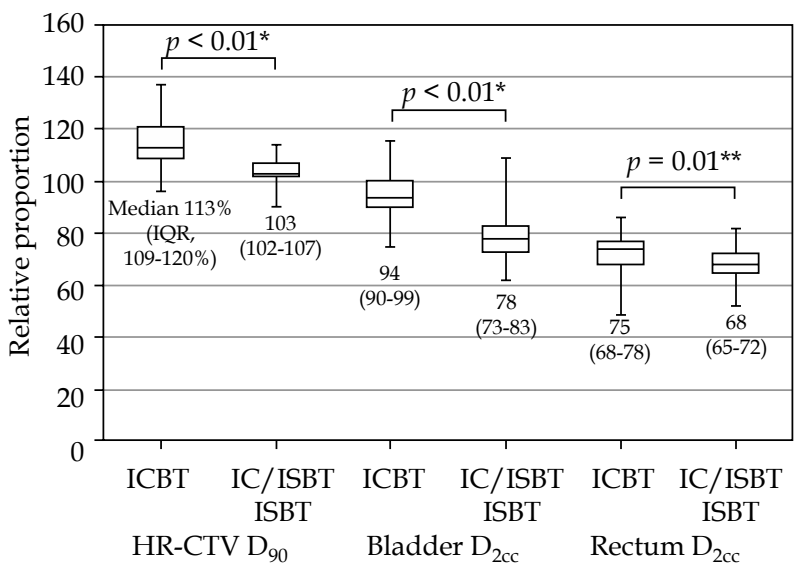

Fig. 5. Relative proportions of clinical target volume (CTV) $\mathrm{D}_{90}$ and organs at risk

$I Q R$ - interquartile range, ICBT - intracavitary brachytherapy, IS/ICBT - combined intracavitary brachytherapy with interstitial technique

*Student t-test, **Mann-Whitney U-test

be selected according to the findings from pre-treatment MRI. Although Palhares et al. reported that large tumor at pre-treatment MRI (not pre-brachytherapy MRI) could predict to deliver inadequate dose to HR-CTV by using ICBT [23], in a previous study, $46-57 \%$ of patients with pre-treatment MRI-evaluated severe vaginal invasion, asymmetrical tumor, or bulky primary tumor received IS/ICBT or ISBT, which were selected according to the findings of pre-brachytherapy MRI; tumor characteristics in pre-treatment MRI were not prognostic factors for LC [10]. Thus, pre-brachytherapy MRI was suitable to select an appropriate brachytherapy method rather than pre-treatment MRI

Recent studies $[5,19,20,21,23]$ demonstrated that tumor characteristics were most likely to benefit from ISBT or IC/ISBT in pre-treatment, pre-brachytherapy MRI, or MRI at the time of brachytherapy; however, type of brachytherapy was not selected according to their criteria before their first brachytherapy. In this study, IS/ICBT or ISBT selected according to the findings in pre-brachyther- 
apy MRI were performed in 26 patients $(43 \%)$, and HRCTV with a mean volume of $62.7 \mathrm{cc}$ (IQR, 46.5-79.7 cc) was administered as the adequate dose (median $\mathrm{D}_{90}$ of 73.7 Gy, IQR, 70.9-75.1 Gy). Among the patients treated with ICBT, there was no significant difference in $\mathrm{EQD}_{2}$ of HR-CTV $\mathrm{D}_{90}$ between HR-CTV volume of $\leq 30 \mathrm{cc}$ and $>30 \mathrm{cc}(p=0.76)$, and ICBT could administer the adequate dose to the tumor, which had no extensive involvement and was larger than $30 \mathrm{cc}$, while providing tolerable doses to the OARs. Therefore, our selection criteria according to pre-brachytherapy MRI findings were effective in selecting suitable candidates for HDR brachytherapy, and might lead to LC improvement also at facilities, where IS/ICBT or ISBT are not routinely performed.

This study's limitations include its single-center retrospective design. Further prospective multicenter studies are needed to evaluate the suitability of selection criteria based on pre-brachytherapy MRI.

\section{Conclusions}

Pre-brachytherapy MRI may help to select suitable candidates for HDR brachytherapy and deliver the recommended doses to tumor and OARs, in particular, in cases with large tumors, severe vaginal invasion, asymmetrical tumors, and/or corpus invasion.

\section{Acknowledgements}

This research was supported by the University of Tsukuba. Statistical analysis was supported by Makoto Saito, MS.

\section{Disclosure}

The authors report no conflict of interest.

\section{References}

1. Landoni F, Maneo A, Colombo A et al. Randomised study of radical surgery versus radiotherapy for stage Ib-IIa cervical cancer. Lancet 1997; 350: 535-540.

2. Goksedef BP, Kunos C, Belinson JL et al. Concurrent cisplatin-based chemoradiation International Federation of Gynecology and Obstetrics stage IB2 cervical carcinoma. Am J Obstet Gynecol 2009; 200: 175.e1-5.

3. Perez CA, Grigsby PW, Nene SM et al. Effect of tumor size on the prognosis of carcinoma of the uterine cervix treated with irradiation alone. Cancer 1992; 69: 2796-2806.

4. Kovalic JJ, Perez CA, Grigsby PW et al. The effect of volume of disease in patients with carcinoma of the uterine cervix. Int J Radiat Oncol Biol Phys 1991; 21: 905-910.

5. Sturdza A, Potter R, Fokdal LU et al. Image guided brachytherapy in locally advanced cervical cancer: improved pelvic control and survival in RetroEMBRACE, a multicenter cohort study. Radiother Oncol 2016; 120: 428-433.

6. Potter R, Georg P, Dimopoulos JC et al. Clinical outcome of protocol based image (MRI) guided adaptive brachytherapy combined with 3D conformal radiotherapy with or without chemotherapy in patients with locally advanced cervical cancer. Radiother Oncol 2011; 100: 116-123.

7. Nag S, Martínez-Monge R, Selman AE et al. Interstitial brachytherapy in the management of primary carcinoma of the cervix and vagina. Gynecol Oncol 1998; 70: 27-32.
8. Wang C, Raince J, Swamy U et al. Clinical outcomes using image-guided interstitial brachytherapy for definitive cervical cancer patients with high-risk clinical target volumes greater than 30 cc. Brachytherapy 2018; 17: 392-398.

9. Murofushi KN, Yoshioka Y, Ishikawa H et al. Selection criteria and clinical outcomes of patients with asymmetrical cervical cancer treated with various high-dose-rate brachytherapy techniques. Anticancer Res 2020; 40: 999-1006.

10. Murofushi K, Yoshioka Y, Sumi M et al. Outcomes analysis of pre-brachytherapy MRI in patients with locally advanced cervical cancer. Int J Gynecol Cancer 2020; 30: 473-479.

11. Rivard M, Coursey B, DeWerd L et al. Update of AAPM Task Group No.43 Report: A revised AAPM protocol for brachytherapy dose calculations. Med Phys 2004; 31: 633-674.

12. Haie-Meder C, Pötter R, Van Limbergen E et al. Recommendations from Gynaecological (GYN) GEC-ESTRO Working Group (I): concepts and terms in 3D image based 3D treatment planning in cervix cancer brachytherapy with emphasis on MRI assessment of GTV and CTV. Radiother Oncol 2005; 74: 235-245.

13. Dale RG. The application of the linear-quadratic dose-effect equation to fractionated and protracted radiotherapy. $\mathrm{Br}$ J Radiol 1985; 58: 515-528.

14. Viswanathan AN, Beriwal S, De Los Santos JF et al. American Brachytherapy Society consensus guidelines for locally advanced carcinoma of the cervix. Part II: High-dose-rate brachytherapy. Brachytherapy 2012; 11: 47-52.

15. Murakami N, Kasamatsu T, Wakita A et al. CT based three dimensional dose-volume evaluations for high-dose rate intracavitary brachytherapy for cervical cancer. BMC Cancer 2014; 14: 447.

16. Lang S, Nulens A, Briot E et al. Intercomparison of treatment concepts for MR image assisted brachytherapy of cervical carcinoma based on GYN GEC-ESTRO recommendations. Radiother Oncol 2006; 78: 185-193.

17. Toita T, Kitagawa R, Hamano T et al. Phase II study of concurrent chemoradiotherapy with high-dose-rate intracavitary brachytherapy in patients with locally advanced uterine cervical cancer: efficacy and toxicity of a low cumulative radiation dose schedule. Gynecol Oncol 2012; 126: 211-216.

18. Ohno T, Noda SE, Okonogi N et al. In-room computed tomography-based brachytherapy for uterine cervical cancer: results of a 5-year retrospective study. J Radiat Res 2017; 58: 543-551.

19. Serban M, Kirisits C, de Leeuw A et al. Ring versus ovoids and intracavitary versus intracavitary-interstitial applicators in cervical cancer brachytherapy: results from the EMBRACE I study. Int J Radiat Oncol Biol Phys 2020; 106: 1052-1062.

20. Jastaniyah $\mathrm{N}$, Yoshida $\mathrm{K}$, Tanderup $\mathrm{K}$ et al. A volumetric analysis of $\mathrm{GTV}_{\mathrm{D}}$ and $\mathrm{CTV}_{\mathrm{HR}}$ as defined by the GEC ESTRO recommendations in FIGO stage IIB and IIIB cervical cancer patients treated with IGABT in a prospective multicentric trial (EMBRACE). Radiother Oncol 2016; 120: 404-411.

21. Mahantshetty U, Gudi S, Singh R et al. Indian Brachytherapy Society Guidelines for radiotherapeutic management of cervical cancer with special emphasis on high-dose-rate brachytherapy. J Contemp Brachytherapy 2019; 11: 293-306.

22. Ohno T, Toita T, Tsujino K et al. A questionnaire-based survey on 3D image-guided brachytherapy for cervical cancer in Japan: advances and obstacles. J Radiat Res 2015; 56: 897-903.

23. Palhares DMF, Marconi DG, Azevedo TL et al. Predicting the necessity of adding catheters to intracavitary brachytherapy for women undergoing definitive chemoradiation for locally advanced cervical cancer. Brachytherapy 2018; 17: 935-943. 